\title{
DERECHO, MORAL, POLÍTICA
}

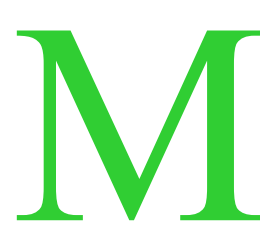
-uchas gracias profesor Fischer. Mi intención, de acuerdo a lo que conversamos previamente, es intentar en el fondo responder a la pregunta que hizo el profesor Atienza en la reunión de ayer y que luego fue intentada responder, también en parte, por el profesor Fisher y por el profesor Duncan Kennedy, y la pregunta es que acá hay dos grupos más o menos definidos de intelectuales; por supuesto cada miembro de ese grupo difiere de otros miembros en aspectos relevantes, pero de alguna manera hay alguna cohesión dentro de estos grupos; y la pregunta es: ¿cómo estos dos grupos difieren? o ¿en qué disentimos? U otra forma de poner la pregunta es: ¿en el fondo no hay más acuerdos de lo que pensamos? Responder a esta pregunta no es fácil, porque supone muchas cuestiones de tipo filosófico general y de tipo filosófico-jurídico en especial y voy a tratar, en un intento inicial y muy provisorio, de aproximarme a una respuesta. Voy a intentar hablar de bastantes temas pero de la forma más esquemática posible. Primero haciendo una caracterización muy tentativa de lo que yo entiendo por método analítico o filosofía analítica y luego una caracterización muy sintética de mi propia visión del Derecho aplicando ese método analítico. La primera cuestión es la cuestión más complicada. Yo no me considero para nada un experto para contestar esta pregunta, pero, en fin, habrá que intentar hacerlo: ¿Qué es el método analítico? ¿Cuáles son los presupuestos o tesis fundamentales de la filosofía analítica? Esto es complejo, hay muy pocos trabajos de metafilosofía en el área analítica y muchos coinciden en que en realidad no hay un dogma analítico, más bien hay una familia de posiciones que se podrían llamar analíticas y son posiciones, más que substantivas, digamos casi procedimentales, en realidad creo que hay muy pocas tesis $[\ldots]^{1}$ y alguna que se podría

${ }^{1}$ La grabación no ha recogido aquí algunas frases. 
considerar como propia de la filosofía analítica: yo creía que a lo mejor una podría haber sido el antiesencialismo en materia conceptual, pero desde la aparición de toda la teoría de las clases naturales y de la teoría semántica de la causalidad de Kripke, Putnam, etc., ni siquiera esa ha quedado en pie como propia de la filosofía analítica. Y por eso es que más bien me animo a decir que el método analítico en principio consiste en hacer una triple distinción -que no es una distinción absoluta, luego hay muchas complicaciones. Pero pretende distinguir entre tres tipos de proposiciones y de actividades de formular estas proposiciones. En primer lugar, lo que podemos llamar el análisis conceptual. Luego, proposiciones de tipo descriptivo. Y finalmente, proposiciones normativas. Todo esto es muy discutible, pero yo creo que un filósofo analítico empieza, por lo menos, distinguiendo estos tres campos y sosteniendo que de lo conceptual no se puede pasar sin solución de continuidad a lo descriptivo o a lo normativo y a la inversa, y, sobre todo, que de lo descriptivo no se puede pasar también sin solución de continuidad a lo normativo. Por supuesto, que hay puentes; hay puentes entre lo conceptual y lo normativo, pero en principio el análisis conceptual es visto como un instrumento que, como todo instrumento, puede en cierta medida ser visto como neutral y en cierta medida no ser visto como neutral. Si uno construye una herramienta, un destornillador, puede hacer muchas cosas, puede hacer cosas buenas o malas; claro, si uno construye un arma esto es menos neutral y hay cierta probabilidad de que con ella haga algo malo. Esto me parece que, a veces, resulta difícil de entender. En mi último curso de Yale (era sobre los derechos sociales) pretendí comenzarlo, como siempre lo hago en Argentina o en España cuando doy cursos de este tipo, con un análisis del concepto de «derecho» en general, que yo pensaba que me iba a tomar media clase, era una cuestión puramente neutra de distinguir diferentes sentidos de la palabra «derecho», etc. Fue imposible. Tardé cinco clases con los estudiantes con este tema, porque ellos creían que había un gato encerrado normativo detrás de mis distinciones conceptuales, o sea que lo que yo estaba haciendo al desarrollar este instrumento era anticipar cuestiones de tipo normativo o justificatorio, lo que no era para nada mi intención. Yo creo que hay algo muy importante acá encerrado. Por supuesto, que un filósofo analítico puede admitir también que haya puentes entre lo descriptivo y lo normativo. En todas las teorías naturalistas o realistas que hay ahora en ética se pretende trazar ese puente. Pero un analítico dirá que el puente no puede ser trazado por cualquier lado: habrá algún puentecito para trazar, pero ¡ojo con la proliferación de puentes! No podemos simplemente, al ir describiendo el mundo, suponer ya que eso implica tomar una cierta actitud hacia ese mundo. En general las 
descripciones del mundo son compatibles con muy diversas actitudes sobre cómo el mundo debe ser cambiado y si debe ser cambiado. Estas distinciones se corresponden -estas dos sobre todo- con una distinción que creo es fundamental para la filosofía analítica y que ya mencioné en una de las reuniones: es la distinción entre razones o justificación y motivos o causación. Esto tiene que ver básicamente con el punto de vista interno y externo frente a cada discurso. Cada discurso tiene reglas o pretende tener criterios que definen qué movidas son razones dentro de ese discurso, qué movidas son razones válidas, y por supuesto esas movidas pueden ser vistas desde fuera del discurso como causadas o determinadas por diversos factores, pero la causación o la determinación o los motivos no son admitidos dentro del discurso como una razón. El hecho de que yo esté diciendo lo que estoy diciendo porque vaya usted a saber qué motivaciones extrañas tengo, que puedan estar relacionadas con mis traumas infantiles, etc., no les interesará a ustedes en esta discusión sobre qué es correcto, etc. A esto, a estas distinciones, corresponde lo que podemos llamar la autoconcepción de la filosofía analítica, cómo se concibe a sí misma la filosofía analítica, qué es lo que hace. Y yo diría que la filosofía analítica se autoconcibe como empezando por un análisis conceptual generalmente muy cuidadoso y preciso, porque se teme -con fundamento, creo yo- que muchísimas discusiones filosóficas, como decía el viejo Austin, se deben a este tipo de confusiones, ambigüedades, vaguedades de los conceptos que estamos empleando; o sea comienza con un análisis conceptual que al principio es relativamente neutral (por ejemplo, el análisis conceptual que hacían el profesor Atienza y el profesor Ruiz Manero, distinguiendo reglas y principios, etc., tiene una cierta neutralidad -digamos- de tipo ético) aunque a medida que uno avanza se empieza a ir a lo que yo creo que es, dentro de la autoimagen, dentro de la autoconcepción de la filosofía analítica, aquello en que, efectivamente, consiste su actividad, que es básicamente una actividad de tipo normativo: es normativa la lógica sobre las reglas del razonamiento correcto y la filosofía de la lógica fundamentalmente; también es normativa en materia epistemológica, esto es, sobre qué es lo que justifica el conocimiento; y es por supuesto normativa en la ética; o sea que el trabajo tal como lo concibe la filosofía analítica, es un trabajo que tiene un énfasis muy importante en el análisis conceptual, en la precisión conceptual, en las definiciones y distinciones de conceptos, pero luego es básicamente un trabajo de tipo justificatorio en distintos ámbitos: en el ámbito del razonamiento, en el ámbito del conocimiento y en el ámbito de las acciones y de las instituciones. Debido a esta distinción entre lo normativo y lo descriptivo se distingue -y acá hay una imagen profesional creo que bastante perfilada 
por parte de la filosofía analítica- entre el trabajo filosófico que es básicamente de análisis conceptual y justificatorio y el análisis de tipo descriptivo, como el que puede hacer un sociólogo o antropólogo, un historiador, etc. (y ambos, desde luego, de otro tipo de tareas como pueden ser las artísticas, literarias, etc.). En esto se pretenden establecer ciertos límites profesionales relativamente definidos.

Pero ahora quiero entrar en la parte más gruesa que es la presentación de mi propia visión del Derecho. Esto fue un intento muy simplificado y esquemático y, además, no hecho por un profesional -digamos- de la metafilosofía, de entender en qué consiste el llamado método analítico, qué presupone la filosofía analítica básicamente. Por supuesto hay muchas complicaciones, cada uno de estos términos tiene muchísimas interpretaciones diferentes, hay muchas posibilidades de establecer conexiones y esto también constituye parte del trabajo de la filosofía analítica, la conexión que pueda haber entre estos términos que aquí hemos visto... Bueno ¿cómo puedo presentar en el menor tiempo posible, para dejar el máximo de tiempo a la discusión, mi visión del Derecho o dar cuenta de la forma en que yo interpreto el Derecho? Creo que lo puedo hacer esquemáticamente también acá mostrando una relación entre tres términos, que son el Derecho, la Moral y la Política. Podría sintetizar lo que pretendo hacer en todos esos trabajos que distribuí como un intento en definitiva de conectar el Derecho con la Política a través de la Moral y para esto tengo que tratar de contrarrestar intentos tradicionales de disociar tanto el Derecho de la Moral, como de disociar la Moral de la Política. La distinción y la disociación entre Derecho y Política es un dogma o una posición importantísima en la historia de las ideas jurídicas. Muchísimas de las ideas jurídicas tradicionales se defienden sobre la base de la disociación que diría es todavía más enfática en la cultura jurídica anglosajona que en la cultura jurídica continental europea, por una serie de razones históricas. De esta disociación entre Derecho y Política dependen cosas fundamentales. Muchas cosas han sido justificadas sobre la base de esta disociación; por ejemplo, «el common law», la idea de que hay un Derecho obligatorio para los jueces separado o aislado en cierto grado del proceso político; por ejemplo, por supuesto, la división de poderes: una buena parte de la división de poderes se pretende justificar sobre la base de la distinción entre Derecho y Política; por ejemplo, el respeto de los derechos fundamentales como un coto cerrado frente al proceso político; o por ejemplo, conectado con los temas anteriores, el control judicial de constitucionalidad, el «judicial review», que también muchas veces se intenta defender sobre la base de la desconexión entre Derecho y Política. Yo creo, como muchos, que esta disociación no se justifica y que hay, en cambio, 
una conexión estrecha entre el Derecho y la Política a través de la Moral; y para eso hay que tomar ciertas posiciones respecto del Derecho, respecto de la Moral y respecto de la Política; y quiero muy esquemáticamente en este tono redondear mi exposición mostrando cuáles son las posiciones sobre estos tres términos que están involucrados en este intento de conectar, en última instancia, el Derecho con la Política. Respecto del Derecho, por supuesto, hay la controversia tradicional entre positivismo e iusnaturalismo. En la defensa del Derecho Natural aparece esta controversia como una controversia sobre la relación entre Derecho y Moral. Lo que yo he intentado hacer en algunos de mis escritos es disolver esta controversia, porque lo que trato de mostrar es que esta controversia en parte responde a una confusión conceptual (y acá viene el tema del análisis conceptual). Lo que la motiva es un esencialismo en materia conceptualista al suponer que hay una sola posible definición de Derecho que para algunos es esencialmente neutra en materia valorativa y para otros es una definición moralizada del Derecho; yo creo que eso es injustificable, porque puede haber definiciones puramente descriptivas de Derecho en ciertos discursos y definiciones de Derecho, digamos, moralizadas o normativas en otro tipo de discursos y que, más que el problema de definición, que es un problema importante como tema preliminar pero que no define de ninguna manera la cuestión, lo que hay de fundamental en la relación entre Derecho y Moral son dos temas centrales (por supuesto que hay muchos otros problemas como bien lo mostró el Prof. Laporta además de estos dos que voy yo a mencionar), que son los temas de justificación y el tema de la interpretación del Derecho. En cuanto a la justificación, la pregunta central que pienso es la pregunta crucial de la Filosofía del Derecho es si las proposiciones jurídicas o normas jurídicas, aunque esto puede distinguirse en ciertos niveles de discurso, constituyen por sí mismas y en forma independiente y autónoma razones para justificar acciones y decisiones. Esta, creo, es la pregunta que tanto ha preocupado, con formas muy diferentes de presentación, a los filósofos del Derecho desde siempre, desde la creación de esta disciplina, y todo por supuesto -acá sí se muestra la importancia de la definición- porque depende de qué quiera decir Derecho y qué entendamos por proposición jurídica. Mi respuesta es que si el Derecho se entiende en términos puramente descriptivos como lo propone el positivismo, la respuesta es negativa. Las proposiciones jurídicas o las normas jurídicas no pueden constituir por sí mismas razones suficientes para justificar acciones o decisiones y el porqué es muy simple: simplemente por la distinción entre lo descriptivo y lo normativo. La imposibilidad de pasar de hechos -porque en definitiva acá se está identificando el Derecho existente, el 
Derecho positivo, con ciertos hechos- a razones que justifiquen acciones o decisiones; y por una serie de razonamientos que también ha hecho Juan Carlos Bayón en su último libro, yo creo que se puede mostrar en forma, digamos, cuasiformal y también de varias maneras informales, que para que las proposiciones jurídicas justifiquen acciones o decisiones hay que acudir necesariamente a principios o proposiciones de tipo moral que son aceptados autónomamente -no por cierta fuente de autoridad- que legitimen las normas jurídicas en cuestión o el proceso de producción de esas normas jurídicas en cuestión. En consecuencia, desde el punto de vista justificatorio (por supuesto que no lo estoy demostrando acá, esto requeriría toda una charla por sí mismo, pero no tengo más remedio que ser dogmático), desde el punto de vista justificatorio, digo, hay una necesaria dependencia del Derecho respecto de la Moral. Desde el punto de vista interpretativo pasa lo mismo porque, aun una vez que identificamos a ciertos materiales, a ciertos actos lingüísticos, a ciertos textos, a ciertas prácticas sociales, como los materiales jurídicos relevantes, la pregunta que cabe hacer es cómo transformamos esos hechos o materiales -que de acuerdo a ciertos principios morales es obligatorio para el juez tener en cuenta- en proposiciones. Porque los jueces no pueden usar directamente esos materiales en sus razonamientos, sino que tienen que usar proposiciones: ¿pero cómo transformamos esos materiales en proposiciones? Traté de mostrar en algunos de esos artículos que ese proceso de transformación tiene unos siete pasos; dos de los cuales son de tipo empírico; otros dos son de tipo lógico; y tres de ellos son de tipo valorativo; o sea que también aquí hay que recurrir a consideraciones extrajurídicas o consideraciones morales en sentido amplio. El paso de fijar el criterio general o los criterios generales de interpretación -por ejemplo acudiendo a las intenciones de los órganos o a las costumbres lingüísticas de la comunidad, etc.- es un paso valorativo. El paso de superar las indeterminaciones semánticas y sintácticas que puede tener el material resultante para llegar así a ciertas proposiciones. Luego vienen las indeterminaciones de tipo lógico, como lagunas, contradicciones, etc., y viene el tercer paso de tipo valorativo consistente en superar esas indeterminaciones lógicas. $\mathrm{O}$ sea, que hay por lo menos tres pasos en el proceso de transformación de los materiales jurídicos relevantes en proposiciones en que intervienen valoraciones de tipo moral en un sentido amplio. Ahora bien, y voy redondeando, siguiendo esto, cuando vemos o resolvemos o pretendemos haber resuelto estos dos problemas, el de la justificación y el de la interpretación, y vemos la dependencia del Derecho de la Moral en estas dos dimensiones, aparece lo que yo llamaría dos paradojas o situaciones de incomodidad: una es la paradoja de la irrelevancia moral del 
Derecho y otra es la paradoja de la indeterminación radical del Derecho. La paradoja de la irrelevancia moral del Derecho viene por el hecho de que, si en todo razonamiento justificatorio como el que hacen los jueces es necesario acudir en última instancia a razones morales para justificar las prescripciones jurídicas, la pregunta es ¿por qué necesitamos esas prescripciones jurídicas? ¿por qué no nos quedamos con las razones morales y se terminó? Porque en definitiva esas razones morales en forma directa o indirecta nos pueden proporcionar una solución para el caso y si necesitamos acudir a ellas, ¿por qué no nos quedamos con ellas?, y esto lleva a lo que uno podría llamar un cierto nihilismo jurídico, la idea de que el Derecho es irrelevante desde el punto de vista justificatorio. Por supuesto que tiene muchas otras funciones, pero desde el punto de vista justificatorio sería irrelevante.

La otra paradoja es la de la indeterminación radical porque, en definitiva, si hacemos abstracción de esos tres pasos valorativos que para muchas concepciones éticas dependerán de preferencias o de la subjetividad de la gente o de sus emociones, etc..., de los materiales jurídicos se podría inferir absolutamente cualquier proposición (todo depende de qué premisas valorativas pongamos en el medio, en cada uno de estos tres pasos); entonces tenemos acá también la indeterminación radical. Para resolver esas paradojas, estas dos paradojas, hay que ir, me parece a mí, al segundo término de esta tríada, que es la concepción de la Moral; o sea que el problema de la justificación y de la interpretación nos llevan del Derecho a la Moral y a la necesaria dependencia entre el Derecho y la Moral. ¿Pero cómo superamos estas paradojas? Y acá yo creo que tenemos que pasar a la Moral. Siendo extremadamente esquemático y sintético, me parece que hay dos trampas que nos tiende en materia moral un cierto individualismo (no de ética substantiva, que yo estoy dispuesto a defender, sino un cierto individualismo, digamos, metaético). Y creo que las dos paradojas se pueden superar si es que superamos, ahora sí en el plano de la Moral, estos dos tipos de individualismo metaético. El primer individualismo metaético tiene que ver con cuál es el objeto de la Moral y el segundo tiene que ver con la forma de conocimiento moral, o sea que se mueve en el plano epistémico. En cuanto al objeto de la Moral, el individualismo metaético está conectado con el llamado individualismo metodológico: es la idea de que siempre, aun en el plano jurídico, las pautas morales se refieren a acciones individuales, que, en definitiva, así como el individualismo metodológico en la ciencia supone que las leyes científicas en última instancia se refieren a acciones individuales, el individualismo metaético, respecto del objeto, supone que los principios morales se refieren a acciones individuales; y esto en buena medida, y en casi toda la medida 
en lo que hace al Derecho, al plano jurídico, creo que es un error, un error que no es nada nuevo (lo ha advertido, por ejemplo, Rawls cuando dice que sus principios de justicia no tiene sentido que se apliquen a acciones individuales, sino que deben referirse a estructuras institucionales, lo ha advertido también por otras razones el utilitarismo de reglas), pero que en el plano jurídico no suele ser advertido. Se supone, por ejemplo, que si uno adopta la posición justificatoria de la interpretación a que me he referido, el planteamiento de un juez sería: bueno, yo tengo que desarrollar una acción individual, estoy sólo en el mundo ¿qué principios morales tengo que aplicar? Pero esto es un error, el Derecho no funciona así. Los objetivos del Derecho, fundamentalmente los objetivos para superar los problemas de cooperación que es una buena función del Derecho, se resuelven no por una suma de acciones individuales, sino que se resuelven por una acción colectiva. El Derecho es una gran acción colectiva que transcurre en el tiempo; es lo que muchos han llamado una «convention», una convención, como Lewis, o una práctica social como el mismo Hart lo ha advertido. Esto es muy importante porque quiere decir que el juez que tiene que decidir un caso, no tiene que decidir como si estuviera sólo en el mundo y tomar la mejor decisión. Tiene que decidir cuál es la mejor decisión desde el punto de vista moral respecto a su contribución a una práctica colectiva que depende de las acciones que se hayan hecho en el pasado y depende de las acciones que se vayan a realizar en el futuro y depende de las expectativas y actitudes de otra gente. Su mejor acción individual puede constituir la peor contribución a la práctica colectiva porque puede mover a esa práctica colectiva en una dirección totalmente inadecuada de acuerdo a esos mismos principios morales que el juez defiende. El juez, en consecuencia, tiene que razonar como lo ha mostrado el utilitarismo de reglas, Rawls, etc., y otra gente, Hare mismo. El razonamiento jurídico justificatorio de un juez se da en un plano escalonado, digamos: un primer nivel en el que el juez tiene que ver si la práctica jurídica en su conjunto es una práctica moralmente justificada de acuerdo a ciertos principios valorativos válidos $\mathrm{y}$, si lo es, o lo es condicionadamente siempre que se la redirija en cierta dirección u otra; luego pasa a un segundo nivel de análisis en que tiene que ver si su acción está o no justificada de acuerdo a esa práctica que a su vez está justificada de acuerdo a los principios morales. Esto ayuda a superar por sí mismo estas dos paradojas porque muestra la relevancia desde el punto de vista justificatorio del Derecho concebido ahora como una práctica o convención social; por supuesto, a diferencia del pensamiento convencionalista y de otros autores (e inclusive yo diría del de Dworkin, que podríamos discutir en qué se diferencia esto del Derecho de la concepción 
interpretativa de Dworkin) yo no creo que la práctica social en que consiste el Derecho sea la última razón justificatoria. Esto, nuevamente, nos haría incurrir en la falacia naturalista de suponer que cierto hecho sirve para justificar acciones o decisiones, pero sí constituye un escalón intermedio; nosotros tenemos que justificar de acuerdo con principios valorativos últimos no acciones individuales, sino prácticas o convenciones y luego las acciones en función de esas prácticas o convenciones.

Entonces éste -digamos- antindividualismo en materia de objeto de la Moral social o intersubjetiva sirve a mi juicio para superar estas dos paradojas. Pero también hay otra alternativa de superación que se combina con la anterior, que tiene que ver con el conocimiento de la Moral, con el plano epistémico de la Moral, porque aquí hay una tradición que supone que si alguien no es subjetivista o emotivista en materia moral en lo que cae, como el iusnaturalismo tradicional, es en una especie de individualismo epistémico en materia moral. Esto es lo que voy a atacar: la idea de que uno llega a principios morales válidos aún en el plano intersubjetivo por su propia reflexión individual aislada. Esta, que es una concepción muy internalizada, inclusive en muchas justificaciones del «judicial review», del control judicial de constitucionalidad, yo creo que es una concepción equivocada. No puedo decir en este momento por qué, porque habría que expandir toda una concepción metaética, pero sí la quiero contraponer con otras dos concepciones y decir dogmáticamente cuál es la que a mí me parece plausible. Entonces tenemos este individualismo que yo creo que es también parte de la paradoja de la irrelevancia moral del Derecho y de la indeterminación radical del Derecho, tenemos la posición opuesta, que es un colectivismo epistémico en materia moral que yo creo que es la posición de Habermas (esto puede ser discutible porque Habermas, debido a que no tiene un trabajo analítico demasiado extenso, siempre es discutible; es difícil saber qué sostiene, y en distintos textos parece sostener diferentes cosas) que parece sostener que la única forma posible de acceder al conocimiento de los principios morales últimos es mediante el proceso de deliberación colectiva, y yo creo que este colectivismo -llamémoslo epistémico- también es cuestionable por razones lógicas porque no puede explicar cuál es el input del discurso moral, qué es lo que uno pretende alegar cuando discute con otros. Si solamente podemos tener alguna posibilidad de conocimiento moral a través del resultado del discurso moral entonces ¿qué es lo que estamos haciendo? ¿estamos expresando actitudes, preferencias subjetivas? Y yo creo que esto sería una descripción incorrecta de la fenomenología del discurso moral. 
En consecuencia, me inclino por una tercera posición que llamo constructivismo epistemológico y que consiste en sostener que si bien hay una prioridad epistémica de la deliberación con otros porque es la mejor forma, aunque de ninguna manera infalible, de llegar a posiciones de imparcialidad, esto no excluye absolutamente algún título de acceso a esas posiciones de imparcialidad, y en consecuencia de conocimiento moral, por vía de la reflexión individual aislada. En consecuencia, hay una prelación epistémica del trabajo colectivo de discusión, pero no hay una exclusión, no quiere decir que el individuo no pueda reflexionar por sí mismo y a veces llegar a principios morales correctos y, sobre esa base, seguir discutiendo aún cuando haya un cierto consenso colectivo y pedir que se reabra la discusión; creo que esta posición es suficientemente débil desde el punto de vista epistémico para que no justifique obrar sobre la base de esa reflexión en el plano de los principios morales intersubjetivos, pero es lo suficientemente fuerte como para justificar seguir discutiendo no obstante el consenso colectivo.

Y, por último, y con esto termino, esto se relaciona a su vez con dos posiciones básicas que se dan respecto de la concepción de la Política y fundamentalmente de la Política democrática; dos concepciones que voy a llamar rápidamente, y esto también es muy discutido, «pluralismo» por un lado y una concepción digamos «deliberativa» por el otro lado, como dos concepciones de la Política. Porque si adoptamos esta concepción pluralista, acá se va a producir también una desconexión entre la Moral y la Política que va a implicar una desconexión entre la Política y el Derecho por más interrelacionada que esté la Moral con el Derecho, o sea que acá hay otra posibilidad de cortar o de tender un puente que en definitiva conduzca de la Política hacia el Derecho. La distinción entre pluralismo y concepción deliberativa de la democracia, para decirlo muy esquemáticamente, creo que depende básicamente de si nosotros pensamos o no que el valor de la democracia depende de su capacidad transformadora de las preferencias de la gente. El pluralismo sostiene que no, que el valor de la democracia es independiente de su capacidad de transformación de las preferencias de la gente y esto lo sostiene en su variante economicista, en su variante utilitarista, en su variante, digamos, elitista pluralista de tipo de Schumpeter, etc. La idea del pluralismo es que por diversos mecanismos, por ejemplo, de equilibrio entre diversos grupos -que para gente como Schumpeter, etc., es lo que da valor a la democracia- o de un mecanismo cuasi de mercado, se obtienen resultados valiosos sin pasar por una transformación de las preferencias de la gente. Ahora, acá hay un punto crucial que es la posibilidad o no de distinguir entre preferencias que podemos llamar personales, como la preferencia por ser abogado, 
por tener un auto, o lo que sea, y las preferencias impersonales, por ejemplo de la preferencia que haya esclavitud, o que no haya esclavitud, o que haya libertad de prensa; y acá es donde se ve la diferencia crucial entre el pluralismo y la concepción deliberativa de la democracia y esto afecta precisamente a la relación entre Política y Moral porque las preferencias impersonales, parte de las preferencias impersonales, son preferencias de tipo moral. El pluralismo tiene dos concepciones acerca de las preferencias impersonales. Una concepción implica dejarlas fuera del juego democrático, pensar que las preferencias éstas son de tipo moral como la esclavitud, la libertad de prensa, etc., y precisamente porque son de tipo moral deben distinguirse de la Política, del juego democrático, y deben dejarse a otros órganos, por ejemplo, a los jueces a través del «judicial review»; esto precisamente coincide con un individualismo epistémico respecto de la Moral. Esta es una posibilidad. La otra posibilidad, en el fondo, es equivalente, y consiste en pensar que el juego de la Política es un juego de composición tanto de preferencias personales como impersonales, pero tratando de llegar a un balance de satisfacción agregativo sin determinar si son válidas o inválidas. Se trata de procurar de satisfacer agregativamente las preferencias de la gente sean de tipo personal o sean de tipo impersonal; en el fondo esto es lo mismo porque acá se está presuponiendo como válido un cierto principio de moral intersubjetiva que es un principio de tipo utilitarista al que se deja, asimismo, fuera del juego de la Política, o sea que en el fondo es lo mismo. Y además esta concepción de la Política no tiene en cuenta la lógica diferente de las preferencias personales y de las impersonales; es una lógica diferente porque las preferencias personales pueden ser agregadas y uno está relativamente satisfecho si se las satisface en parte sin discutir la validez de esas preferencias. Yo no quiero que discutan si es bueno o no ser abogado, quiero que se la ponga en la bolsa de las preferencias; pero con las preferencias impersonales pasa lo contrario: uno no pretende que la pongan en la bolsa común y que, bueno, que haya un poquito de esclavitud si yo quiero esclavitud y un poco de libertad si hay otros que quieren libertad; uno pretende que se defina su validez y en el caso de definir su validez que se las satisfaga absolutamente; es lo que Owen Fiss en The Death of Law, pero no hablando de los críticos, sino del análisis económico del Derecho, llama el carácter imperialista de este tipo de preferencias que implican la exclusión de las preferencias opuestas: debe determinarse la validez. Esto lleva a una concepción deliberativa de la democracia. ¿Qué es una concepción deliberativa? Es una concepción por supuesto conectada con el constructivismo epistémico en materia moral y es la concepción según la cual la democracia tiene un poder transformador de las preferencias 
de tipo impersonal (no de las de tipo personal) y en consecuencia la democracia tiene un cierto valor epistémico (dados una serie de mecanismos que son discutibles y que he tratado de defender, etc.) respecto de la validez de las preferencias de tipo impersonal que se constituyen a través del método democrático.

Esta concepción deliberativa de la democracia en la forma que yo la defiendo deja fuera del proceso democrático otras dos cosas: primero las condiciones del mismo proceso democrático, que es lo que llamo «derechos a priori» y que creo que se determinan -y acá hay una analogía con el método kantiano trascendental- a través de analizar las condiciones del conocimiento a través del método democrático: esto queda fuera del método democrático mismo porque son las condiciones que dan validez a ese método democrático; y también quedan afuera precisamente por esas mismas condiciones, las preferencias de tipo personal, de planes de vida, etc., pues no creo que el método democrático respecto de ellas tenga más valor epistémico acerca de su validez, si son válidas o no, que la reflexión individual. Bueno, con esto termino, creo que de esta forma se logra efectivamente trazar este puente y creo que éste es uno de los puntos, me parece, de coincidencia fundamental, por lo que yo entiendo, respecto de la escuela crítica del Derecho: la existencia de esta conexión esencial entre Derecho y Política. No sé. Posiblemente los puentes sean diferentes y posiblemente el rol intermediario de la Moral en esto sea diferente pero creo que el objetivo por lo menos fundamental de conexión entre Derecho y Política se cumple.

Posiblemente, dado lo que traté de explicar al principio, ello se obtenga por métodos diferentes, pero mi objetivo no es tratar de coincidir a toda costa, sino tratar de mostrar (no exactamente, pero sí aproximadamente) cuáles son nuestras diferencias $\mathrm{y}$, tal vez, cuáles son nuestras coincidencias. Gracias. 